Murray Stuart (Orcid ID: 0000-0002-5588-2915)

Griffiths Scott (Orcid ID: 0000-0002-6366-3560)

Lavender Jason (Orcid ID: 0000-0001-9853-2280)

Males Special Issue Editorial

\title{
Introduction to a Special Issue on Eating Disorders and Related Symptomatology in Male Populations
}

\author{
Stuart B. Murray, Ph.D. ${ }^{\text {a }}$, Scott Griffiths, Ph.D. ${ }^{\text {, }}$ \& Jason M. Lavender, Ph.D., ${ }^{\text {c, }}{ }^{*}$ \\ ${ }^{a}$ Department of Psychiatry, University of California, San Francisco, San Francisco, CA, USA \\ ${ }^{\mathrm{b}}$ Melbourne School of Psychological Sciences, University of Melbourne, Melbourne, VIC, \\ Australia \\ ${ }^{\mathrm{c}}$ Department of Medicine, Uniformed Services University of the Health Sciences, Bethesda, \\ MD, USA \\ ${ }^{d}$ Military Cardiovascular Outcomes Research Program (MiCOR), Uniformed Services \\ University of the Health Sciences, Bethesda, MD, USA
}

*Correspondence: Jason M. Lavender, Ph.D., Department of Medicine, Uniformed Services University of the Health Sciences, 4301 Jones Bridge Road, Bethesda, MD 20814-4712. Email: jason.lavender.ctr@usuhs.edu

Keywords: men; males; disordered eating; eating disorder; eating pathology; muscularity; muscularity-oriented disordered eating; body image; treatment; assessment Acknowledgements and Disclosures: Dr. Murray is supported by the National Institute of Mental Health (K23 MH115184), and receives royalties from Routledge and Oxford University Press. The opinions and assertions expressed herein are those of the authors and do

This is the author manuscript accepted for publication and has undergone full peer review but has not been through the copyediting, typesetting, pagination and proofreading process, which may lead to differences between this version and the Version of Record. Please cite this article as doi: $10.1002 /$ eat.23184

This article is protected by copyright. All rights reserved. 
not necessarily reflect the official policy or position of the Uniformed Services University or the Department of Defense.

\title{
RUNNING TITLE: MALES SPECIAL ISSUE EDITORIAL
}

\author{
Abstract \\ Objective: It is our sincere pleasure to introduce a special issue of the International \\ Journal of Eating Disorders focused on eating disorders and related symptoms in \\ male populations.
}

Method: Contributions focused on three main themes among male populations: (i) the nature of eating disorder symptoms and recovery; (ii) long-term outcomes following eating disorders treatment; and (iii) muscularity-oriented symptoms of eating pathology.

Results: The contributions to this special issue highlight the importance of better understanding how eating disorder symptoms manifest, may be maintained, and respond to treatment among male populations. Findings across the articles demonstrate some similarities between male and female samples with respect to core eating disorder symptoms, as well as important differences, particularly with respect to muscularity-oriented disordered eating. Findings related to treatment outcome also highlight the suboptimal long-term remission rates and elevated mortality among male inpatients with eating disorders.

This article is protected by copyright. All rights reserved. 
Discussion: The diverse range of samples, methodologies, and research questions addressed within this collection of papers demonstrates the breadth of the important scientific work on this topic. As eating disorders and related symptoms in male populations continue to be underrecognized and underserved, we hope that this special issue will encourage further high-quality clinical research in this area.

This article is protected by copyright. All rights reserved. 
We are delighted to introduce a special issue of the International Journal of Eating Disorders focused on eating disorders and related symptomatology in male populations. Historically, eating disorders have been broadly conceptualized from a female-centric perspective, and prior diagnostic frameworks have reflected this (e.g., amenorrhea as a former criterion for anorexia nervosa; APA, 2000). Importantly, evidence indicates that boys or men with eating pathology may be less likely than girls or women to seek treatment; and when male patients do present for treatment, their eating disorder may go undetected or receive less intense intervention compared to female patients (Forrest, Smith, \& Swanson, 2017; Striegel-Moore, Leslie, Petrill, Garvin, \& Rosenheck, 2000; Striegel, Bedrosian, Wang, \& Schwartz, 2012; Thapliyal, Hay, \& Conti, 2018). Further, empirical research over several decades commonly omitted boys and men from controlled treatment trials on the basis of supposed atypicality, and to date, less than $1 \%$ of all eating disorder- and body imagerelated research has focused exclusively on male populations (Murray et al., 2017). Cumulatively, this has resulted in the under-recognition of eating disorder psychopathology among boys and men, and propagated the misunderstanding that eating disorders rarely impact this population. Such misunderstandings likely contribute to the stigma of eating disorder symptoms in male populations, which in turn may interfere with help-seeking among boys and men struggling with eating pathology (Andersen \& Holman, 1997; Griffiths, Mond, Murray, \& Touyz, 2015; Räisänen \& Hunt, 2014). 
With evidence indicating that eating disorder psychopathology (i) is increasingly prevalent among male populations, (ii) is comparable in severity to that demonstrated in female populations, and (iii) may be qualitatively distinct from symptom profiles typically reported in female populations, the need for more focused research with boys and men is clear (Lavender, Brown, \& Murray, 2017; Lewinsohn, Seeley, Moerk, \& Striegel-Moore, 2002; Murray et al., 2017; Smith et al., 2017; Striegel-Moore, Garvin, Dohm, \& Rosenheck, 1999; Thapliyal, Mitchison, Mond, \& Hay, in press). To that end, this special issue represents a significant step forward in advancing our collective understanding of this understudied area, and offers important advances relating to the conceptualization, measurement, and outcomes of/recovery from eating disorder and related psychopathology in male populations.

\section{Identifying Symptom Dynamics and Defining Recovery in Men}

Network theory and analytic approaches provide an innovative conceptual and statistical framework for the study of psychiatric disorders, including eating disorder psychopathology (Smith et al., 2019). Contrasting with traditional conceptualizations suggesting that symptoms of mental disorders arise from underlying syndromes, network theory posits that psychiatric illnesses such as eating disorders emerge from systems of interacting symptoms (Borsboom, 2017). Using a network analytic approach, Perko and colleagues (2019) examined sex differences in systems of eating disorder psychopathology using sex-specific network analyses and a subsequent series of network comparison tests. The authors utilized data collected via the Recovery Record app, with men comprising half of the sample. Findings indicated that the 
structure of eating disorder psychopathology networks was similar for men and women, with binge-eating emerging as the symptom most central to the network. The authors concluded that eating disorders in men and women may be maintained through similar processes, although it must be noted that certain eating disorder symptoms, including those relating to muscularity-oriented symptoms, were not assessed and thus were not available for inclusion within the network analyses. This study represents a promising advance in our understanding of eating disorder symptom structure and dynamics across men and women, and will hopefully encourage and guide future research based on this novel and informative approach. In addition to elaborating the experience and presentation of eating disorder symptoms, understanding the nature of recovery from eating disorders among male populations is also crucial. The definition of eating disorders recovery remains contentious and the majority of research in this area has been conducted with female samples (Bardone-Cone, Hunt, \& Watson, 2018). Given evidence suggesting sex differences in eating disorder symptoms across various cognitive, behavioral, and physiological domains, there is an important need to consider how definitions of recovery originally based on female populations might apply similarly or differently in male samples. In addressing this gap, Bardone-Cone and colleagues (2019) examined whether their previously published definition and operationalization of eating disorders recovery (Bardone-Cone et al. 2010), developed and validated among women, was also appropriate for men. The authors compared men with no lifetime history of eating disorders to men with a current eating disorder and men in either full 
or partial recovery. Incorporating physical, behavioral and cognitive elements, the authors concluded that recovery for men involves reductions in expectancies about thinness and dietary restriction, reductions in body shame, greater ease stopping negative thoughts about one's body, diet and exercise, and better attitudes about one's level of both muscularity and body fat. Encouragingly, men that had fully recovered from eating disorders did not differ from men with no lifetime history of an eating disorder. The authors also concluded that, small sample size notwithstanding, their definition of eating disorders recovery usefully applies to men.

\section{Treatment Outcomes in Males}

Data addressing eating disorder treatment outcomes in male populations have been limited, since boys and men have historically been excluded from clinical trials, or represented only a small minority of those included. As such, the application of clinical interventions with male populations rests on inferences drawn from research conducted with largely female samples. However, in the largest prospective study to date of treatment outcomes in males with eating disorders, Strobel and colleagues (2019) and Quadflieg and colleagues (2019) describe a series of important outcomes in a large sample of male inpatients with eating disorders over an average follow-up period ranging from approximately five to nine years across the diagnostic groups. In assessing long-term treatment outcomes among 119 men with anorexia nervosa and 60 men bulimia nervosa, Strobel and colleagues (2019) noted that males with anorexia nervosa demonstrated comparable rates of remission to females with anorexia nervosa treated in the same program (male remission rate: $40 \%$, female 
remission rate: $41 \%$ ), and typically demonstrated greater body weight. Among male inpatients with bulimia nervosa, remission rates were slightly lower at long-term follow (44\%) relative to female inpatients treated in the same program (50\%). However, male inpatients with bulimia nervosa reported relatively lower scores on measures of eating disorder psychopathology at follow-up. Cumulatively, these data offer promising insights into eating disorders treatment outcomes in male populations. With regard to mortality rates, the elevated mortality associated with eating disorders is well established (Fichter \& Quadflieg, 2016; Smink, van Hoeken, \& Hoek, 2012). However, data on mortality specifically among males with eating disorder psychopathology has been sparse. In their study, Quadflieg and colleagues (2019) found that standardized mortality ratio (SMRs) were significantly elevated in male inpatients diagnosed with anorexia nervosa, and results of survival analyses also revealed shorter long-term survival after eating disorder onset for anorexia nervosa compared to both bulimia nervosa and eating disorder-not otherwise specified. These results highlight the seriousness of eating disorders in male populations, particularly those with anorexia nervosa, and thus further support the importance of appropriate identification and treatment of boys and men with eating disorders to improve longterm outcome and reduce mortality.

\section{Muscularity-Oriented Disordered Eating in Men}

Boys and men face societal pressures to conform to an idealized body type characterized by a muscular and lean build, and increasingly girls and women may also face pressures related to these dimensions of body shape (Murray et al., 2017; 
Rodgers et al., 2018). Pursuing muscularity and striving to increase lean mass may promote a unique array of disordered eating symptoms that differ from those associated with the traditional pursuit of thinness (Lavender et al., 2017). Given that adolescence and young adulthood is a time of particular risk for onset and/or exacerbation of eating pathology, muscularity-oriented disordered eating behaviors may also be particularly salient within this age range (Calzo et al., 2016). Using nationally representative prospective data from the Longitudinal Study of Adolescent to Adult Health, Nagata and colleagues (2019) examined baseline adolescent predictors of muscularity-oriented disordered eating during young adulthood at 7-year follow-up. Nearly one quarter of young men and one in twenty young women reported any muscularity-oriented disordered eating behavior at the follow-up. Further, numerous demographic, behavioral, and physiological baseline factors were identified as significant predictors of muscularity-oriented disordered eating for both young adult men and women, whereas other behavioral and mental health predictors were significant only for one sex. These findings highlight the importance of identifying youth at greater risk for disordered eating behaviors of various forms, and support the potential utility of prevention or early intervention.

The findings from Nagata and colleagues (2019) also support the need for novel assessment measures that incorporate a broader variety of eating pathology symptoms. Specifically, the majority of self-report assessments of disordered eating were historically developed and validated with female samples, and were based on a traditional conceptualization of eating pathology consistent with pursuit of the thin 
body ideal. However, efforts to achieve a muscular and lean body, which characterizes the modern ideal male body, may involve distinct types of disordered eating behaviors. The article by Murray and colleagues (2019) describes the development and initial psychometric validation of a new measure of muscularityfocused disordered eating: the Muscularity-Oriented Eating Test (MOET). This twostudy article reports the initial development of the measure in Study 1, which derived a 15-item, one-factor measure that was confirmed in Study 2. Findings provide preliminary evidence supporting the internal consistency and test-retest reliabilities of the MOET score, as well as convergent validity based on moderate to large associations with relevant measures of eating pathology, muscle dysmorphia, and drive for muscularity. Building on other recent efforts to improve on the assessment of eating pathology in boys and men, the MOET has potential utility in both research and clinical contexts as a novel measure of muscularity-oriented disordered eating.

\section{Conclusion}

It was pleasure to oversee this special issue on eating disorders and related symptomatology in male populations. Given the relative dearth of rigorous empirical research addressing this area, we hope that this focused collection of manuscripts will encourage greater recognition of these issues among boys and men, as well as foster further ideas and high-quality scientific work with this population. We would like to thank all authors who shared their valuable contributions, and we look forward to the continued growth of this important literature within the eating disorder field. 


\section{References}

Andersen, A. E., \& Holman, J. E. (1997). Males with eating disorders: challenges for treatment and research. Psychopharmacology Bulletin, 33, 391-397.

American Psychiatric Association. (2000). Diagnostic and Statistical Manual of Mental Disorders, fourth edition, text revision (DSM-IV-TR). American Psychiatric Publishing.

Bardone-Cone, A. M., Harney, M. B., Maldonado, C. R., Lawson M. A., Robinson, P., Smith, R., \& Tosh, A. (2010). Defining Recovery from an Eating Disorder: Conceptualization, Validation, and Examination of Psychosocial Functioning and Psychiatric Comorbidity. Behaviour Research and Therapy, 48, 194-202.

Bardone-Cone, A. M., Hunt, R. A., \& Watson, H. J. (2018). An Overview of Conceptualizations of Eating Disorder Recovery, Recent Findings, and Future Directions. Current Psychiatry Reports, 20, 79.

Bardone-Cone, A. M., Johnson, S., Raney, T. J., Zucker, N., Watson, H. J., \& Bulik, C. M. (2019). Eating disorder recovery in men: A pilot study. International Journal of Eating Disorders.

Borsboom, D. (2017). A network theory of mental disorders. World Psychiatry, 16, 513.

Calzo, J. P., Horton, N. J., Sonneville, K. R., Swanson, S. A., Crosby, R. D., Micali, N., ... Field, A. E. (2016). Male Eating Disorder Symptom Patterns and Health Correlates From 13 to 26 Years of Age. Journal of the American Academy of Child and Adolescent Psychiatry, 55, 693-700.

This article is protected by copyright. All rights reserved. 
Fichter, M. M., \& Quadflieg, N. (2016). Mortality in eating disorders - results of a large prospective clinical longitudinal study. International Journal of Eating Disorders, 49, 391-401.

Forrest, L. N., Smith, A. R., \& Swanson, S. A. (2017). Characteristics of seeking treatment among U.S. adolescents with eating disorders. International Journal of Eating Disorders, 50, 826-833.

Griffiths, S., Mond, J. M., Murray, S. B., \& Touyz, S. (2015). The prevalence and adverse associations of stigmatization in people with eating disorders. International Journal of Eating Disorders, 48, 767-774.

Lavender, J. M., Brown, T. A., \& Murray, S. B. (2017). Men, muscles, and eating disorders: an overview of traditional and muscularity-oriented disordered eating. Current Psychiatry Reports, 19, 32.

Lewinsohn, P. M., Seeley, J. R., Moerk, K. C., \& Striegel-Moore, R. H. (2002). Gender differences in eating disorder symptoms in young adults. International Journal of Eating Disorders, 32, 426-440.

Murray, S. B., Brown, T. A., Blashill, A. J., Compte, E. J., Lavender, J. M., Mitchison, D., ... Nagata, J. M. (2019). The development and validation of the muscularity-oriented eating test: A novel measure of muscularity-oriented disordered eating. International Journal of Eating Disorders.

Murray, S. B., Nagata, J. M., Griffiths, S., Calzo, J. P., Brown, T. A., Mitchison, D., ... Mond, J. M. (2017). The enigma of male eating disorders: A critical review and synthesis. Clinical Psychology Review, 57, 1-11. 
Nagata, J., Murray, S. B., Bibbins-Domingo, K., Garber, A. K., Mitchison, D., Griffiths, S. (2019). Predictors of muscularity-oriented disordered eating behaviors in U.S. young adults: A prospective cohort study. International Journal of Eating Disorders.

Perko, V. L., , Forbush, K. T., Siew, C., \& Tregarthen, J. (2019). Application of network analysis to investigate sex differences in interactive systems of eating-disorder psychopathology. International Journal of Eating Disorders.

Quadflieg, N., Strobel, C., Naab, S., Voderholzer, U., \& Fichter, M. M. (2019). Mortality in males treated for an eating disorder-A large prospective study. International Journal of Eating Disorders.

Räisänen, U., \& Hunk, K. (2014). The role of gendered constructions of eating disorders in delayed help-seeking in men: a qualitative interview study. $B M J$ Open, 4, 4.

Rodgers, R. F., Franko, D. L., Lovering, M. E., Luk, S., Pernal, W., \& Matsumoto, A. (2018). Development and Validation of the Female Muscularity Scale. Sex Roles, 78, 18-26.

Smink, F. R. E., van Hoeken, D., \& Hoek, H. W. (2012). Epidemiology of Eating Disorders: Incidence, Prevalence and Mortality Rates. Current Psychiatry Reports, 14, 406-414.

Smith, K. E., Crosby, K. E., Wonderlich, S. A., Forbush, K. T., Mason, T. B., \& Moessner, M. (2018). Network analysis: An innovative framework for 
understanding eating disorder psychopathology. International Journal of Eating Disorders, 51, 214-222.

Smith, K. E., Mason, T. B., Murray, S. B., Griffiths, S., Leonard, R. C., Wetterneck, C.T., ... \& Lavender, J. M. (2017). Male clinical norms and sex differences on the Eating Disorder Inventory (EDI) and Eating Disorder Examination Questionnaire (EDE-Q). International Journal of Eating Disorders, 50, 769775.

Striegel, R. H., Bedrosian, R., Wang, C., \& Schwartz, S. (2012). Why men should be included in research on binge eating: results from a comparison of psychosocial impairment in men and women. International Journal of Eating Disorders, 45, 233-240.

Striegel-Moore, R. H., Garvin, V., Dohm, F. A., \& Rosenheck, R. A. (1999). Psychiatric comorbidity of eating disorders in men: a national study of hospitalized veterans. International Journal of Eating Disorders, 25, 399-404. Striegel-Moore, R. H., Leslie, D., Petrill, S. A., Garvin, V., \& Rosenheck, R. A. (2000). One-year use and cost of inpatient and outpatient services among female and male patients with an eating disorder: evidence from a national database of health insurance claims. International Journal of Eating Disorders, 27, 381-389.

Strobel, C., Quadflieg, N., Naab, S., Voderholzer, U., \& Fichter, M. M. (2019). Longterm outcomes in treated males with anorexia nervosa and bulimia nervosa- 
A prospective, gender-matched study. International Journal of Eating Disorders.

Thapliyal, P., Hay, P., \& Conti, J. (2018). Role of gender in the treatment experiences of people with an eating disorder: a metasynthesis. Journal of Eating Disorders, 6, 18.

Thapliyal, P., Mitchison, D., Mond, J., \& Hay, P. (in press). Gender and help-seeking for an eating disorder: findings from a general population sample. Eating and Weight Disorders.

This article is protected by copyright. All rights reserved. 


\section{University Library}

\section{- M M N E R VA A gateway to Melbourne's research publications}

Minerva Access is the Institutional Repository of The University of Melbourne

Author/s:

Murray, SB;Griffiths, S;Lavender, JM

Title:

Introduction to a special issue on eating disorders and related symptomatology in male populations

Date:

2019-10-14

\section{Citation:}

Murray, S. B., Griffiths, S. \& Lavender, J. M. (2019). Introduction to a special issue on eating disorders and related symptomatology in male populations. INTERNATIONAL JOURNAL OF EATING DISORDERS, 52 (12), pp.1339-1342. https://doi.org/10.1002/eat.23184.

Persistent Link:

http://hdl.handle.net/11343/286496 